\title{
Typologie des sécheresses sur la France et outils de suivi de la ressource en eau utilisés à Météo-France
}

\section{BLANCHARD, \\ L. FRANCHISTÉGUY}

Météo-France.

Direction de la Climatologie

42, ave $G$. Coriolis

31057 Toulouse Cedex michele.blanchard@meteo.f

F. HABETS

CNRSUMMR-Sisyphe 7619 Paris

\section{É MARTIN, J. NOILHAN CNRM-GAME (Météo-France, CNRS)} Toulouse
La France a subi des sếcheresses en 2003, 2005 et 2006 , dont les conséquences notamment sur les bătiments ont mis en lumière l'importance de ce risque naturel.

En raison de ses missions, Météo-France suit l'ensemble des phénomènes météorologiques, dont les sécheresses, via la quantification des déficits pluviométriques observés par un important réseau de mesure de précipitations et des systèmes d'analyse atmospherique. Dans cet article, on présente les différents types de sécheresse et la comparaison des sécheresses récentes par rapport aux sécheresses connues dans le passé. De plus, Météo-France utilise des modèles de suivi du bilan en eau comme le modèle Safran Isba Modcou [SIM] pour mieux appréhender les conséquences des déficits pluviométriques et de la demande évaporative sur l'extension et l'intensité de la sécheresse. SIM pernet de calculer des bilans d'eau spatialisés et en particulier un indice d'humidité du sol et un stock de neige sur les montagnes. Ces données sont utilisées dans le suivì hydrologique en France. Les caractéristiques et quelques résultats en terme de contenu en eau du sol de deux de ces modèles sont présentés.

Mots-clés : sécheresse, ressources en eau, hydirométéorologie, changement climatique.

dryness, via the quantification of the pluviometric deficits based on an important observation network of precipitations and some atmospheric analysis. In this article, the various types of dryness and the comparison of the recent drynesses compared to the older ones are presented. Météo-France also monitors the water budget by using models as the Safran Isba Modcou [STM] model. Such model allows to estimate the consequences of the pluvionetric deficits and the evaporative demand on the soil water content, and thus, on the extersion and the intensity of the dryness. The characteristics and some resuts in term of soil moisture from two of these models are presented. SIM allows calculating assessments of water and in particular indication of humidity of the ground and stock of snow on mountains. These data are used in the bydrological follow-up in France.

Key words: drought, water resources, hydrometearology, climate change. 


\section{Introduction}

La sécheresse géotechnique est la conséquence visible sur le bâti de mouvements de terrain liés au changement de volume du sol (principalement argileux) subissant un assèchement inhabituel. Cette notion de sécheresse géotechnique est donc proche de la notion de sécheresse, mais, restreinte plus particulièrement à certains types de sol (argileux et pentus par exemple). Dans cet article, on s'intéressera à la notion de sécheresse, dans le sens générigue du terme, qui peut ểtre définj de la manière suivante : la sécheresse correspond au maintien sur des périodes de longueur variable (de la semaine à plusieurs années) de conditions hydriques déficitaires par rapport à un seuil critique (état normal, maximal ou nécessaire au bon développement de la végétation).

La sécheresse se caractérise donc par un déficit de précipitation, dont l'effet peut être accentué par une forte demande évaporative de !'atmosphère. MétéoFrance est particulièrement engagé dans les actions de suivi de sécheresse. Il a en effet pour mission le suivi des cumuls de précipitations, à partir d"un réseau d'observation (pluviomètres et radars) assez étendu, d'analyse et aussi de prévision. De plus, Météo-France, via les modèles de prévision du temps et de climat, a acquis une expertise sur la modélisation du bilan hydrique en surface. Cette expertise est utilisée depuis plusieurs années pour le suivi opérationnel du bilan hydrique, qui est géré en temps réel, et permet notamment de répondre à des besoins sur l'estimation de la ressource en eau, pour l’agriculture, et pour la prévision des feux de forêts.

Dans cet article, on présente tout d'abord les causes météorologiques conduisant à une sécheresse, les différents types de sécheresse ainsi que celles ayant le plus marqué par leur amplitude ou leur intensité. Ensuite, on présente les outils utilisés pour réaliser le suivi de la ressource en eau en temps réel, et quelques résultats obtenus dans la demière décennie. Enfin, on aborde l'impact attendu du changement climatique sur les sécheresses, dans le monde et en France.

\section{2}

\section{Causes et localisation}

Les sécheresses permanentes concernent les zones arides soumtses à des manques de précipitation chroniques. Ces zones sont en général des régions continentales éloignées des océans (on ne traitera pas de ce type de sécheresse par la suite). Les sécheresses de durée limitée se chiffrant en semaines ou en mois peuvent être observées pratiquement partout ailieurs et notamment en France métropolitaine.

Les sécheresses de durée limitée sont en général liées à des anomalies de circulation atmosphérique de grande échelle favorisant les hautes pressions tant au sol qu'en altitude. De ce fait, il s'agit toujours de phénomènes s'étendant sur plusieurs centaines de $\mathrm{km}^{2}$ (F"ig. 1). Par ailleurs, des phénomènes de grande échelle comme celui d'El Niño qui modifie la circulation de l'océan Pacifique interfère par exemple sur la circulation atmosphérique et provoque des conditions arides en Indonésie, en Inde ou en Australie.
En France, les causes de sécheresse sont souvent liées à la persistance d'un anticyclone qui rejette les perturbations vers le nord.

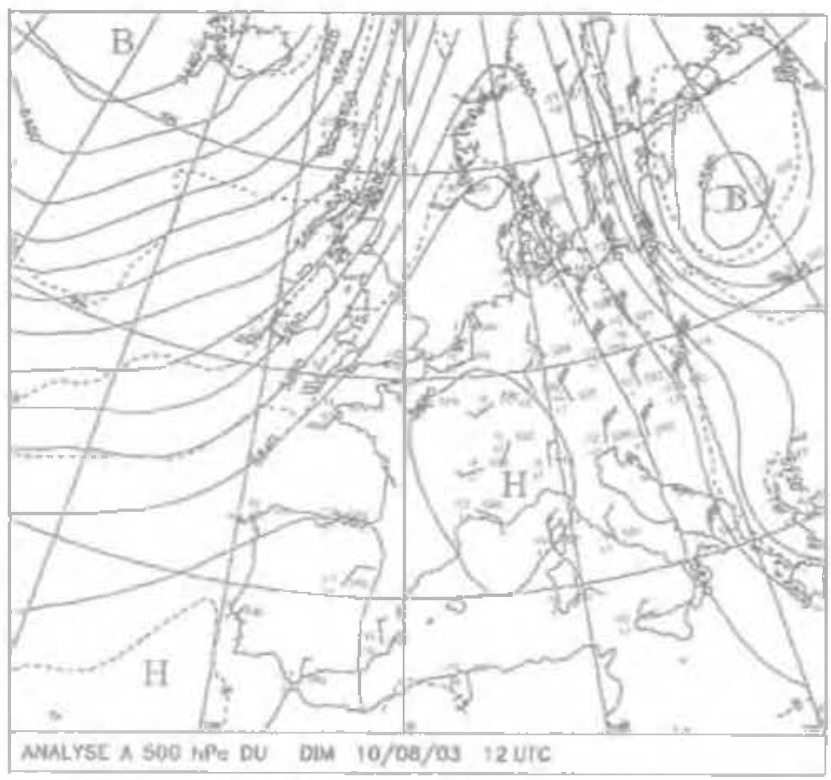

FG. 1 Situation météorologique type provoquant des épisodes de sécheresse marqués.

Typical meteorological situation prowoking marked episodes of drought.

\section{3}

\section{Types de sécheresse} tants :

On distingue les sécheresses, selon les effets résul-

- la sécheresse de l'atmosphère correspondant à un déficit d'humidité de l'air, résultant de l'absence de précipitations, d'une évaporation lou évapotranspiration) limitée, du fait de contenus en eau du sol et/ou de la végétation réduits ou insuffisamment mobilisables; elie est le premier maillon d'un ensemble de processus qui affectent les milieux naturels et les activités humaines;

- la sécheresse du sol superficiel ou profond (nappes phréatiques), lée à des précipitations déficitaires, à une évaporation importante ou à un raissellement de surface important. C'est ce type de sécheresse qui affectera les constructions

- la sécheresse de la végétation, liée à un déficit d’hydratation des tissus végétaux, résultant d"une alimentation en eau insuffisante de la plante.

L'effet de la sécheresse dépend de sa durée et de son intensité, de la periode où elle intervient, mais aussi des réserves en eau du sol el du stade de développement des végétaux :

- la sécheresse hivernale affectera en priorité le remplissage des nappes phréatiques :

- la sécheresse printanière ou estivale aura un fort impact sur la végétation et les miliewx vivants aquati ques:

- les sécheresses pluriannuelles cumuleront ces effets et auront ainsi un fort impact tant écologique qu'économique. 


\section{Les sécheresses historiques}

A l'étranger, les évènements extrêmes les plus souvent cités font état de 3 à 10 millions de morts en Inde (1769-1770), 9,5 millions de morts en 1877-1879 en Chine, 100000 morts dans l'état du Ceara au Brésil l'été suivant, 5 millions de morts en Russie (1921-1922), 3 millions de morts au Bengale en 1942-1943 et plus près de nous de 400000 victimes au Sahel dans les années $1970-1980$

Parmi les grandes sécheresses que la France a connues au $X X^{\circ}$ siècle, celle de 1976 est particulièrement exceptionnelle par sa durée (décembre 1975-août 1976) et par son intensité (l'été 1976 a été l'un des plus secs du siècle). Elle a surtout touché la moitie nord-ouest du pays. De décembre 1975 à aout 1976. les précipitations mensuelles ont été majortairement défjcitaires sur la plupart des régions. Pour certains mois tels que février et juillet, les écarts à la nomale n'étaient pas très importants ; par contre, décembre 1975, puis janvier, maj et surtout juin 1976 ont présenté des prẻcipitations particulèrement faibles. La sécheresse intense s'est poursuivie au mois d'août dans la moitié nord du pays, alors que la moitié sud était touchée par des pluies fortement excédentaires. Le tableau I fournit des valeurs du rapport à la normale des pluies du 1/12/1975 au 31/7/1976 pour quelques stations représentatives.

Pour cette période. Brochet (1976) a estimé en étudiant des séries centenaires sur 25 sites et en calculant les quantiles de durée de retour moyen, qu'un rapport pluviométrique $<0,4$ avait une durée de retour supếrieure à 100 ans, $<0,5$ une durée de retour supérieure à 50 ans, <0,6 une durée de retour supérieure à 20 ans. Bien sûr, la diversité des régimes pluviométriques ne permet pas d'appliquer ces résultats sur une région particulièrement.

La sécheresse de 1989 (qui a démarré dès août 1988 pour s'achever en novembre 1989) a surtout frappé le sud et le sud-est, avec une amplitude comparable à celle de 1976. L'hiver 1988/1989 a été remarquable par son manque de neige en montagne.

A la canicule de l'été 2003 a été associée une sécheresse estivale sévère qui a affecté tout particulièrement les bâtis. Les températures élevées, tant diurnes que noctumes, ont nettement aggravé les effets du déficit pluviométrique.

Plus récemment, la sécheresse a affecté le centre et la façade ouest du pays durant les années 2005 et 2006 mettant à mal le remplissage de grandes nappes phréatiques comme celle de la Beauce.

Le tableau II indique quelques sécheresses en France au siècle dernier.

\section{5}

\section{Suivi de la sécheresse à Météo- France}

L'apport en eau par les précipitations et l'état hydrique du sol constituant des facteurs communs pour les différents types de sécheresse, les météorologistes coractérisent la sécheresse en utilisant ces deux facteurs, en particulier pour déterminer la nature exceptionnelle de certaines périodes.

Afin d'assurer un suivi de la ressource en eau sur La France, la direction de l'Eau (DE) du ministère de l'Écologie, de l'Aménagement et du Développement durable réalise mensuellement un bulletin de situation hydrologique national. Météo-France contribue à ce bulletin en alimentant les rubriques « eau dans le sol "et " manteau neigeux ) avec des sorties du modèle hydrométéorologique SIM (Safran-Isba-Modcouj et par la fourniture de données permettant une cartographie des précipitations et des précipitations efficaces ainsi que Ieur rapport à la nornale. La DE a, par ailleurs, confié à l'établissement l'élaboration de la partie météorologique des bulletins de situation hydrologique régionaux avec la fourniture de cartes et de données pour les paramètres précipitations, pluies efficaces et évapotranspiration potentielle. Durant la période d'étiage, Météo-France réalise un bulletin décadaire de suivi de la sécheresse et contribue aux comités sécheresse locaux el nationaux. Une expertise est aussit assurée sur les dossiers de catastrophes naturelles inondations et sécheresse soumis à la cornmission Catastrophes naturelles qui est présidée par la direction de la Défense et de la Sécurité civiles.

TAblzau I Valeurs du rapport à la normale des pluies du 1/12/1975 au 31/7/1976 pour queiques stations représentatives. Values of the report in the normal of the tains of $1 / 12 / 1975$ in 31//1976 for some representative stations.

\begin{tabular}{l|l|l|l}
\hline Abbeville & 0,40 & Bourges & 0,59 \\
\hline Lille & 0,47 & Nevers & 0,44 \\
\hline Cherbourg & 0,36 & Limoges & 0,69 \\
\hline Beauvais & 0,37 & Clermont-Fertand & 0,62 \\
\hline Reins & 0,43 & Lyon & 0,64 \\
\hline Chartres & 0,39 & Grenoble & 0,53 \\
\hline Paris Orly & 0,45 & Bourg-St-Maurice & 0,43 \\
\hline Langres & 0,39 & Agen & 0,56 \\
\hline Metz & 0,58 & Bordeaux & 0,69 \\
\hline Strasbourg & 0,62 & Toulouse & 0,57 \\
\hline Le Mans & 0,44 & Nîmes & 1,05 \\
\hline Lorient & 0,38 & Marseille & 1,35 \\
\hline Brest & 0,44 & Nice & 0,86 \\
\hline Rennes & 0,46 & Ajaccia & 0,84
\end{tabular}


zunu II Quelques sécheresses en France au siècle dernier. Some droughts in France in the last century.

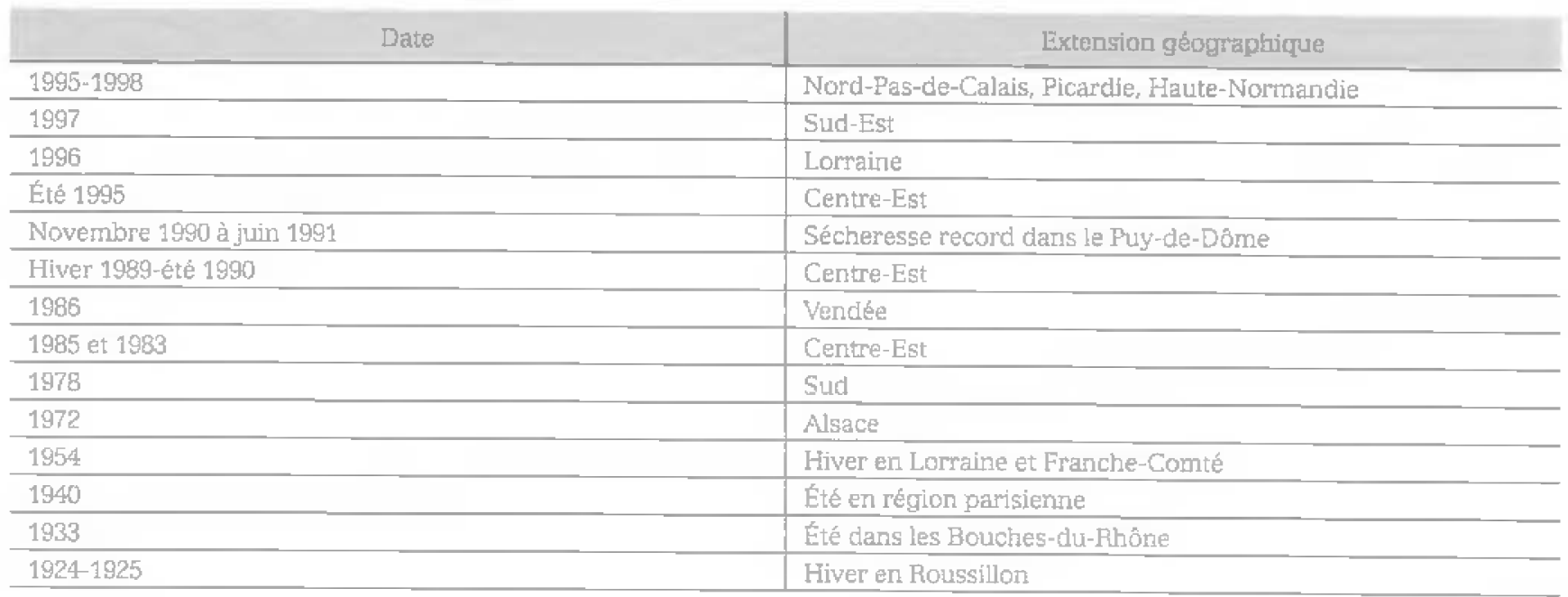

\section{6}

\section{Outils de suivi de la sécheresse à Météo-France}

Météo-France utilise depuis plus de dix ans des outils de modélisation permettant de suivre I'impact des conditions climatiques sur le contenu en eau du sol, et donc, ainsi, sulvre et quantifier l'état de sécheresse. Un premier modèle, d'origine agronomique, a tout d'abord êté utilisé. Ce modèle est utilisé sur un certain nombre de points représentatifs, et ne prend en compte que l'impact sol-végétation-atmosphère. Depuis environ cinq ans, un deuxième modèle a été implémenté. Ce modèle permet de couvrìr l'ensemble de la Frarce et permet de simuler, en plus des relations sol-végétation-atnosphère, le débit des rivières et le niveau de certaines nappes phréatiques. De plus, la couverture neigeuse, qui peut avoir localement un rôle important sur le bilan hydrique, est prise en compte explicítement. Ces deux modèles sont présentés ci-dessous.

\section{1}

\section{Modèle de bilan hydrique à deux réservoirs}

Le modèle simplifié (Jacquart et Choisnel, 1995) est couramment utilisé à Météo-France pour des besoins agrométéorologiques avec des rẻserves utiles variant de 50 à $200 \mathrm{~mm}$ selon lé type de couvert végêtal et/ou la profondeur des sols. On considère qu'en pratique, trois valeurs de réserve utile convenablement choisies permettent de couvrir l'éventail des réserves utiles rencontrées pour les types de sol d'un département.

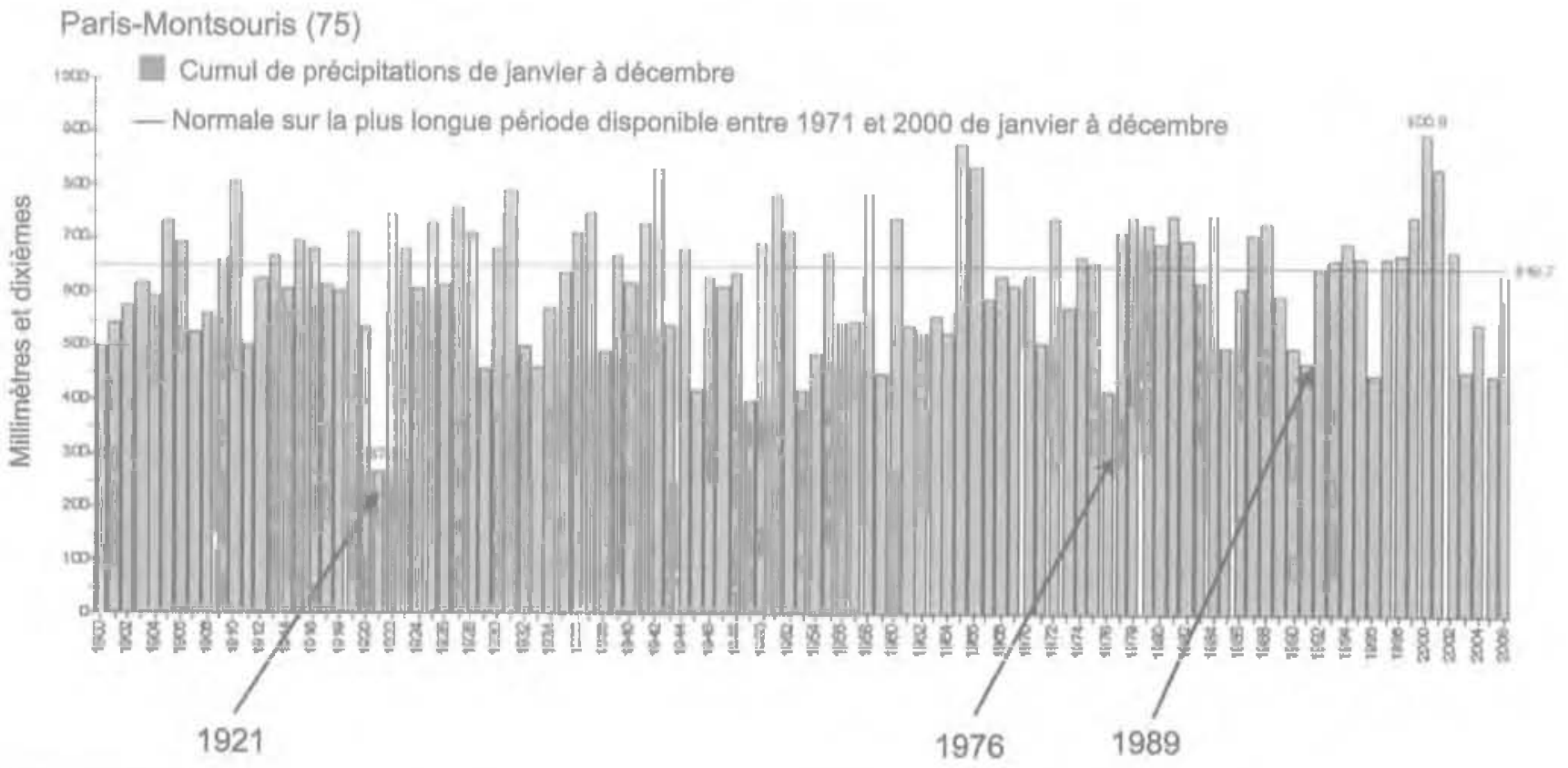

Fí. Cumul annuel de pluie sur le poste Paris-Montsouris sur la période 1900-2006. Annual rainy accumulation on the Paris-Montsouris station over the period 1900-2006. 
Le principe du modèle est de considérer que c'est par l'intermédiaire du système racinaire du couvert végétal considéré que l'eau est extraite du sol (mécanisme d'évapotranspiration]. Le réservoir de surface est évaporé au taux potentiel pondéré par le coetficient cutural. Le réservoir profond n'est évaporé cuiune fois le réservoir de surface vide, à un taux diminuant avec son contenu (réponse linéaire par rapport ả l'état du réservoir) (Fìig. 3).

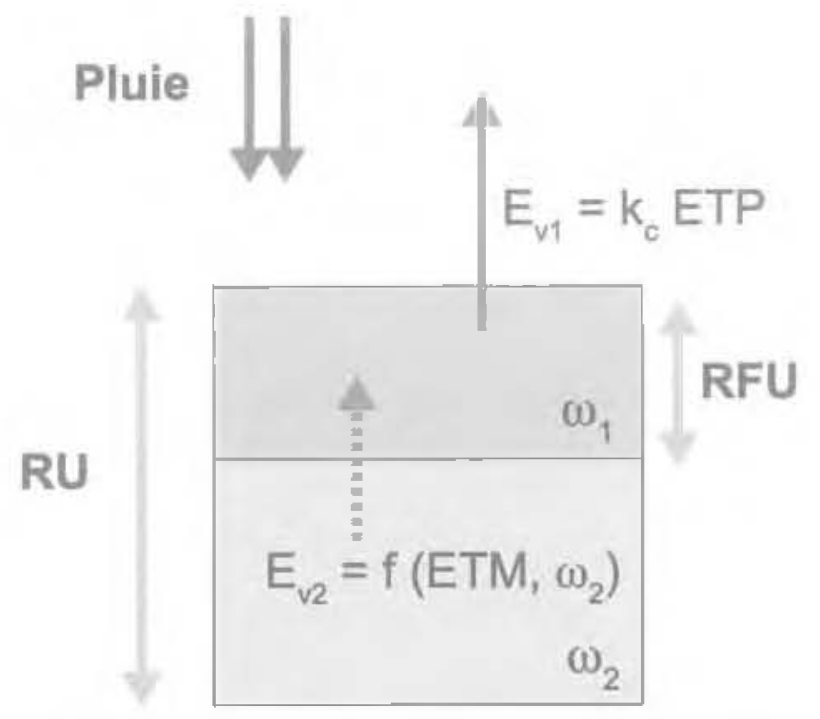

$\mathrm{RU}=$ réserve utile $\quad \mathrm{RFU}=$ réserve facilement utile

Principe du modéle de bilan hydrique à deux réservoirs.

Principle of the madel of assessment hydrique of 2 reservoirs.
L'écoulement (qu'il soit ruissellement de surface ou écoulement profond) n'est pas distingué et se produit lorsque la réserve calculée dépasse la réserve utile.

On considère que l'eau profonde située au-delà de la limite des racines les plus profondes n'est pas disponible en surface (les remontées capillaires étant fałbles). Ceci a par exemple été confirmé par les mesures d"humidité des sols lors de l'expérience Murex près de Toulouse, sol argileux couvert par une jachère vieille de plusieurs années. On a bien constaté que l'huntcité du sol au-delà de $120 \mathrm{~cm}$ restait pratiquement constante au cours de l'année. Le gazon est la couverture de surface de ce modèle. Cette méthode ne rend pas compte des caractérstiques pédologiques du sol, si ce n'est par le biais simplifié de la réserve utile. La neige, le gel dans le sol ainsi que les bilans énergétiques et thermiques du sol ne sont pas intégrés. En revanche, ce modèle robuste et à l'approche agronomique a l'avantage de se focaliser sur une gamme de réserve utile commune à l'erisemble des sites simulés.

Dans ce modèle, le calcul du bilan hydrique s'effectue sur les postes du réseau de Météo-France et est conditionné par la disponibilité des paramêtres nécessaires à ce calcul (environ 140 postes à ce jour). Pour chaque poste, le calcul est effectué avec des réserves utiles de $50,100,150$ et $200 \mathrm{~mm}$. La profondeur du réservoir superficiel est fixée à $40 \%$ de la réserve utile. Ce modèle effectue un bilan à l'aide des précipitations et des évapotranspirations (ETP) quotidiennes puis simule les échanges entre le réservoir superficiel et le réservoir profond avec un 'frein' appliqué à la récupération de l'eau stockée dans le réservoir profond.

La figure 4 présente les résultats obtenus par ce modèle en terme d'évolution du réservoir d'eau disponible sur la période 1995-2004 et en moyenne sur l'ensemble des postes disponibles en France.

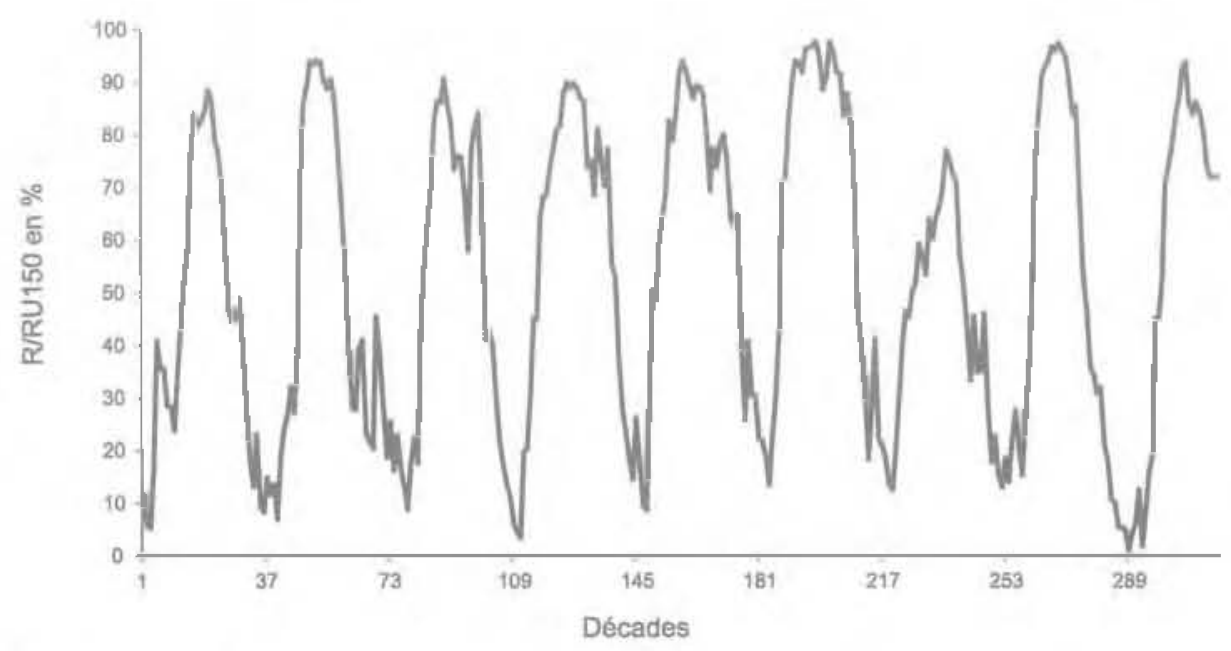

月a. 4 Moyenne du rapport entre le contenu en eau du réservoir et la réserve utile (R/RU) pour une réserve utile de $150 \mathrm{~mm}$. Les résultats sont moyennés sur I'ensemble des postes disponibles sur la France, sur la période 1995-2004. 


\section{Modèle hydrométéorologique Safran-Isba-Modcou (SIM)}

\author{
6.8.1 \\ Principe
}

SIM (Habets et al., 1999a ; Habets et al., 2007) est un modèle hydrométéorologique constitué de l'assemblage de 3 modèles, qui permet d'estimer les bilans d'eau et d'énergie en surface, sur I'ensemble de la France, à une résolution spatiale de $8 \mathrm{~km}$, ainsi que le niveau de plusieurs nappes souterraines et les dẻbits des rivières à plus de 900 postes. Ce système initialement développé sur les bassins de l'Adour, du Rhône. de la Garonne et de la Seine a été étendu sur la France en 2002 puis mis en opérationnel en 2003.

Ce modèle repose sur l'enchaînement de trois éléments : Safran, Isba et Modcou.

Les variables atmosphériques (précipitations, température, rayonnements solaire et infrarouge, vent et humidité de l'air) sont analysées pari le système SAFRAN (Durand et al., 1992), qui prend en compte l'ensemble des observations disponibles, des analyses de modèle opérationnel, et des données climatiques, pour produire des cartes de 8 variables atmosphériques au pas de temps horaire, et à une résolution de $8 \mathrm{~km}$.

Le module ISBA (Interactions-Sol-BiosphèreAtmosphère, schéma de surface utilisé ici dans sa version hydrologique à 3 couches, (Noilhan et Planton, 1989; Noilhan et Mahfouf, 1996; Boone et al., 1999) calcule les bilans d'eau et d'énergie sur l'ensemble solvégétation, en prenant en compte la composante nivale (très importante sur les massifs montagneux), au pas de temps de 5 minutes. Ge schéma est en outre utilisé dans les modèles opérationnels de prévisions atmosphériques de Météo-France. ISBA prend en compte de manière explicite les paramétres de texture de sol (base des sols de I'INRA a $1 \mathrm{~km}$ ) et le type de végétation (base ECOCLIMAP, Masson et al., 2003, modifiée sur la France pour distinguer les types de cultures ayant différents cycies annuels, voir figure 5].

Enfin, le module MODCOU (Ledoux et al., 1989), développé par l'École des mines de Paris, simule le niveau des nappes, leur interaction avec les rivières, et les débits quotidiens sur pius de 900 postes en France.

\section{a.9.9.}

\section{Suivi du bilan hydrique estimé par SIM}

Les simulations de SIM sont réalisées en double: une première analyse est réalisée en temps a quasi réel (traitement quoticien de la période J-1, 6h à J, 6h), et utilise les observations disponibles sans délaí. Ainsi, l'analyse quotidienne "temps réel' de précipitations utilise approximativement 1200 à 1300 postes de mesures pluviométriques par jour sur le territoire. Une deuxième analyse est réalisée en temps différé $(\mathrm{J}+45$, donc avec plus d'un mois de délai), afin de collecter l'ensemble des données du réseau climatologique d'êtat. Dans cette analyse, on dispose alors de plus de 3000 postes pluviométriques.

Cela permet de constituer une base de données des paramètres météorologiques interpolés par Safran (une critique détaillée de ces analyses a été réalisée par Quintana Segui et al., 2007) mais aussi des tormes du bilan hydrique, constituant ainsi une référence pour une analyse plus pertinente des situations du temps présent. Actuellement, l'archive historique du modèle déterminée en utilisant le réseau climatologique complet couvre la période début août 1970 à 2007

Ainsi, les cumuls de précipitations totales (liquide et neigeuse) analysés par Safran permettent de situer une année donnée par rapport à la climatologie existante. La figure 6 présente la comparaison des cumuls annuels de précipitations pour la normale 1971-2000, et pour les cinq années sèches suivantes : 1976, 1989, 1990,2003 et 2004. Il apparaît clairement que les

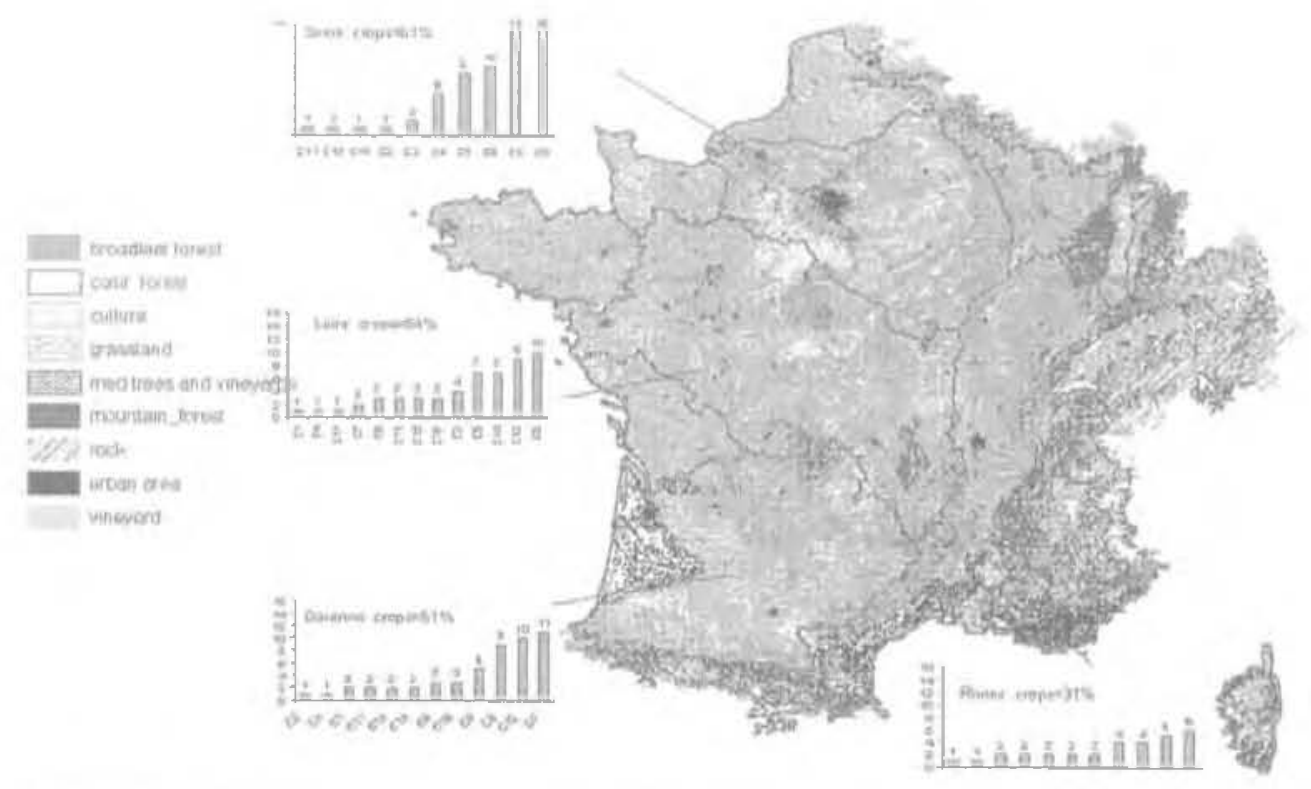

Fe. 5 Carte d'occupation des sols Ecoclimap sur la France.

Soil type map Ecoclimap on France. 


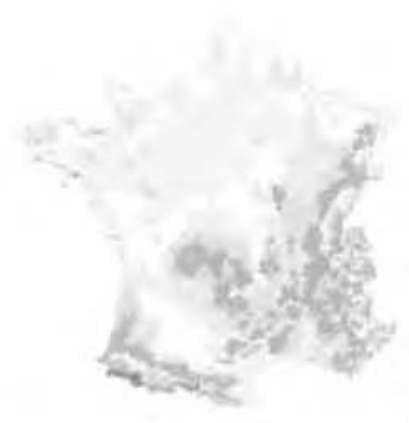

1976

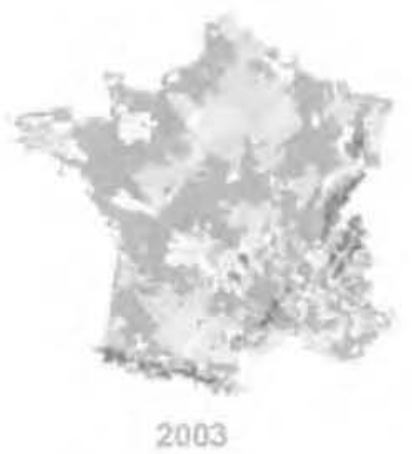

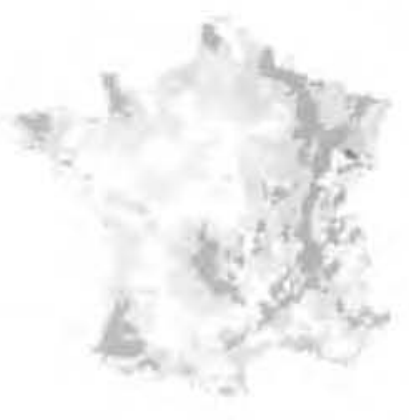

1989

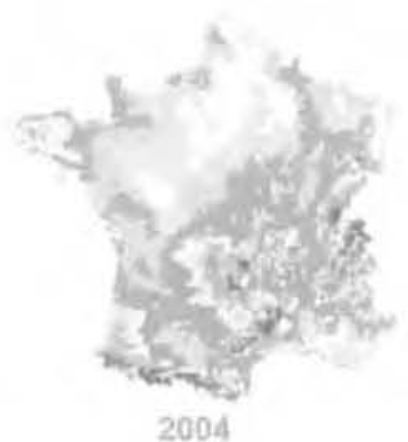

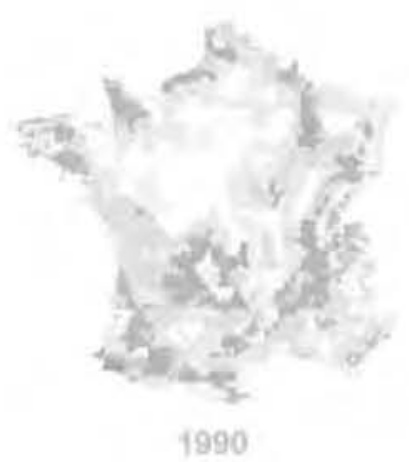

di

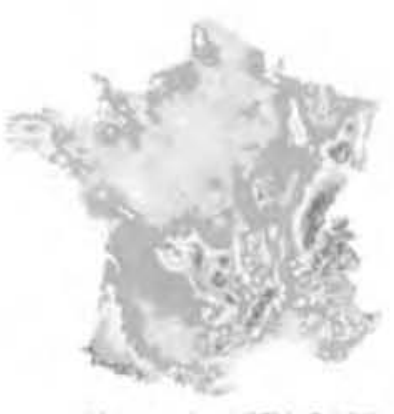

Normale 1971-2000

Fa. 6 Cumul annuel de précipitations Safran sur la France d'août à juillet et comparaison à la nor'male Aurelhy sur la période 1971-2000.

déficits de précipitations des années sèches varient beaucoup à la foís en intensité et dans l'espace.

Les termes du bilan hydriques simulés par SIM peuvent être partiellement validés, grâce à la comparaison entre les simulations et les observations de débits, de niveaux piézométriques et de hauteurs de neige. Ainsi, une analyse des résultats de SIM sur la décennie 19952005 a montré que l'évaporation annuelle était bien estimée, avec une errewr moyenne sur l'historique disponible de l'ordre de $4 \%$ du flux d'évaporation annuel (Habets et al., 2007). Cette erreur varie d'une année sur l'autre avec un maximum de l'erreur de $8 \%$ pour l'année 2000-2001 sur le bassin du Rhône. Cela permet d'avoir une bonne confiance dans le bilan hydrique simulé par SDM.

Ansi, on peut s'intéresser aux évolutions temporelle et spatiale du contenu en eau du sol.

Pour étudier les variabilités spatiales du contenu en eau du sol, on utilise l'indice d'humidité des sols SWI (Soil Wetness Index) défini par :

avec:

$$
S W I=\left(W-W_{\text {wilt }}\right) /\left(W_{i c}-W_{\text {wilt }}\right)
$$

W = contenu intégré en eau du sol ;

$W_{\text {wit }}=$ contenu en eau du sol au point de flétrissement, seuil à partir duquel la plante ne peut plus capter l'eau du sol, trop rare ;

$W_{\text {fc }}=$ contenu en eau du sol à la capacité au champ. seul au-dessus duquel il n'y a plus de drainage gravitationnel dans le sol.

Lorsqu'il est au-dessus de 1 , le sol est humide, et les plantes ne sont pas stressées. En dessous de 1, il y a stress hydrique, d'autant plus sévère que l'indice se rapproche de 0. L'évolution moyenne sur la France de l'indice d'humidité des sols simulée par SIM sur la période 1995-2005 est présentée figure 7. On constate que l'évolution temporelle de l'indice d'humidité des sols issu de SIM sur la France se compare bien à la moyenne décadaire du pourcentage de remplissage du réservoir (R/RU à $150 \mathrm{~mm}$ ) simulé par le modèle à deux réservoirs présenté figure 4 . En particulier, on observe que les valeurs minimales sur cette période sont atteíntes durant l’année 2003, suive de l'année 1998.

Indice d'humidité moyen

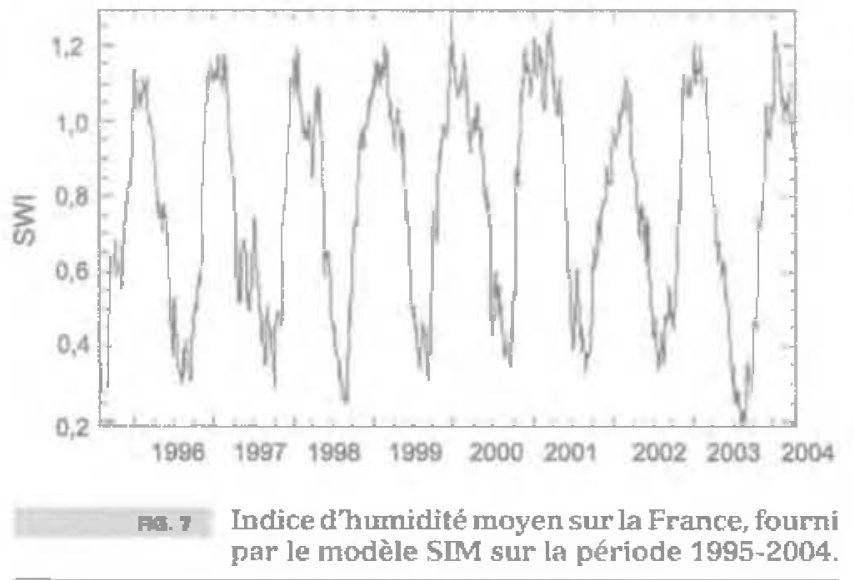

L'indice d'humidité des sols est estimé quotidiennement ainsi que l'écart par rapport à la climatologie du modèle SIM disponible sur la période 1995-2006. 
Une telle estimation pour le début du mois de juin 2006 est présentée figure 8. La carte des écarts à la moyenne est très contrastée, avec une large zone bien excédentaire sur le Nord et l'Est du pays, et de nombreuses petites zones légèrement excédentaires. Le reste du pays est déficitaire. On note deux grandes régions présentant des déficits importants : une large bande allant de la Vienne à la Marne et surtout une grande partie du sud-ouest et de la bordure méditerranéenne, ainsi que la Corse. Ces résultats actualisés sont publiês régulièrement sur le site Internet de MétéoFrance alnsi que dars le bulletin national de situation hyorologique du ministère de l'Écologie de l'Aménagement et du Développement durable.

Aftr de suivre l'état du bilan hydrique sur l'ensemble de la France, on utilise des courbes présentées figure 9. Elles représentent l'évolution temporelle de l'indice d'humidité des sols en moyenne sur l'ensemble de la France sur l'arnnée étudiée (trait épais noir). comparée à la moyenne sur la climatologie 1970-2005 (trait fin noir), ainsì qu'aux valeurs extrèmes (en vert) et aux quintiles $20 \%$. Lorsque la courbe rouge atteint la courbe verte inférieure, cela signifie qu'a ce moment là, l'année étudiée est la plus sèche sur les 35 années étudiées. Quand elle atteint la courbe pointillée, cela siơnifie que le niveau de sécheresse a été atteint 1 année sur 5 en moyenne. Ainsi, on constate que la sécheresse de 2003 a conduit en juillet et en août aux valeurs d'indice d'humidité des sols les plus faibles en moyenne sur la France par rapport à la climatologie sur 35 ans et que des valeurs assez proches avaient déjè été atteintes en 1989 et 1990 .

Le sưvi du bilan hydrique est complêté par le suivi du mantea neigeux. En effet, la fonte du manteau neigeux au printemps apporte un afflux d'eau, qui s'il est important, pourra atténuer l'effet d'une sécheresse, et même dans certains cas contribuer à des inondations.

On peut avoir une bonne confiance dans la représentativité du manteau neigeux simulé par SIM, car les hauteurs de neige simulées ont êté comparées aux nombreuses observations disponibles à différentes altitudes (Etchevers et al., 2001 ; Voirin-Morel, 2003 ; Habets et al., 2007). Les biais sont faibles (inférieurs à 3 $\mathrm{cm}$ pour les bandes altitudes infér l'ordre de $10 \mathrm{~cm}$ au-delà), et les variabilités journalières sont bien représentées (le coefiticient de corrélation au carré est de l'ordre de 0,7 pour les tranches d'altitudes supérieures à $750 \mathrm{~m}$ ).

SIM estime la quantité d'eau stockée sous forme de neige (c'est l'équivalent en eau de la neige). La figure 10 présente la comparaison de ce stock au $1^{\mathrm{er}}$ avtil 2006, et en moyenne sur la décennie 1995-2005 pour la même date dans les Alpes. Le stock d'eau est globalement équivalent à la moyenne des simulations pour cette date sur la période 1995-2005. La quantité d’eau stockée dans le manteau neigeux au 1er avril 2006 n’est légèrement supérieure à la moyenne que sur les Alpes suisses.

\section{7}

\section{Conclusion et perspectives : changement climatique et sécheresse géotechnique}

Le suivi des sécheresses et des ressources en eau effectué par Météo-France se base de plus en plus sur la chaine Safran-Isba-Modcou, opérationnelle depuis quelques années. Cet outil fournit une simulation spatialisée de l'humidité des sols basée sur les bilans couplés hydriques et énergétiques en surface. Le suivi des variables hydrologiques est affiné dans le temps et l'espace, ce qui permettra de mieux répondre aux demandes des autorités dans le domaine.

Les efforts actuels portent sur la reconstitution d'une climatologie de Safran-Isba-Modcou sur une longue période (remontant jusqu'en 1970, et si possibla jusqu'en 1958). On espère mieux caractériser la variabilité inter annuelle des différents paramètres, et qualifier les périodes de sécheresses futures par comparajson avec les événements marquants du passé, comme 1976,1989 ou 2003.
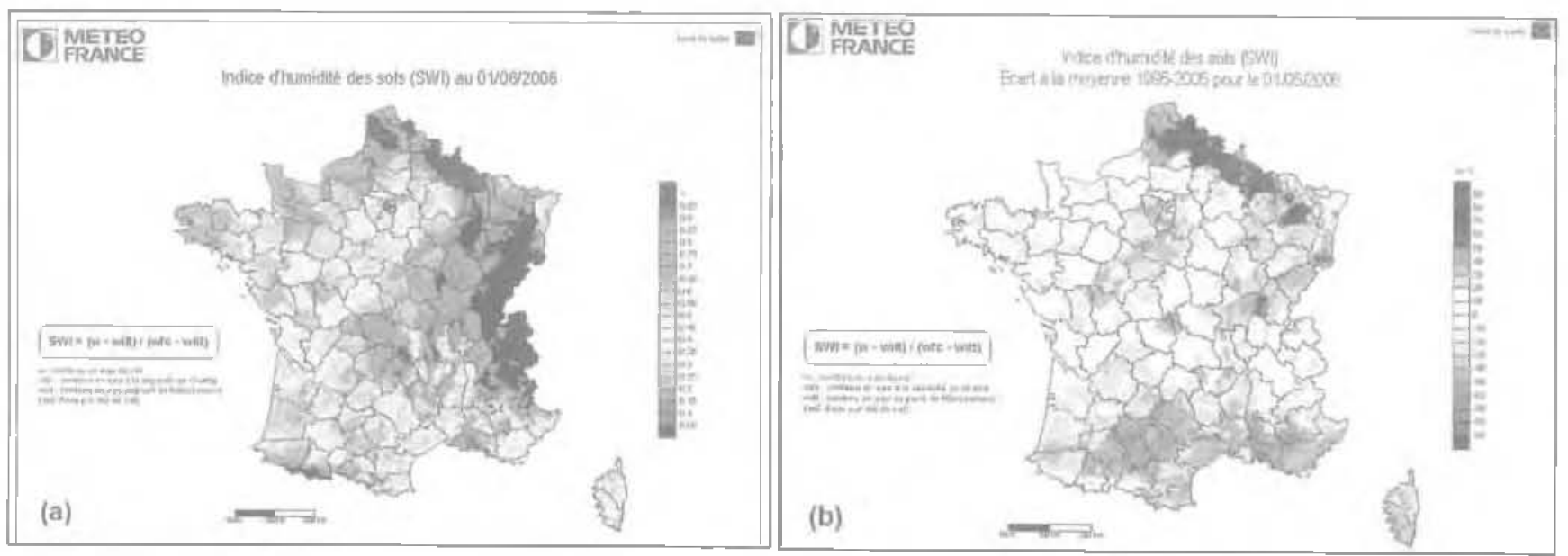

ma. : Contribution de Météo-France à la rubrique ressources en eau du BSH national : (a) indice d"humidité de sol sur la France au 1er juin 2006; (b) écart à la référence 1995-2005 de l'indice d'humidité des sols.

Contribution of Météo-France to the column water ressources of the national BSH: (a ) Soil wetness index over France on June 1st, 2006, ( b ) Anomaly with 1995-2005 climatology of SM 

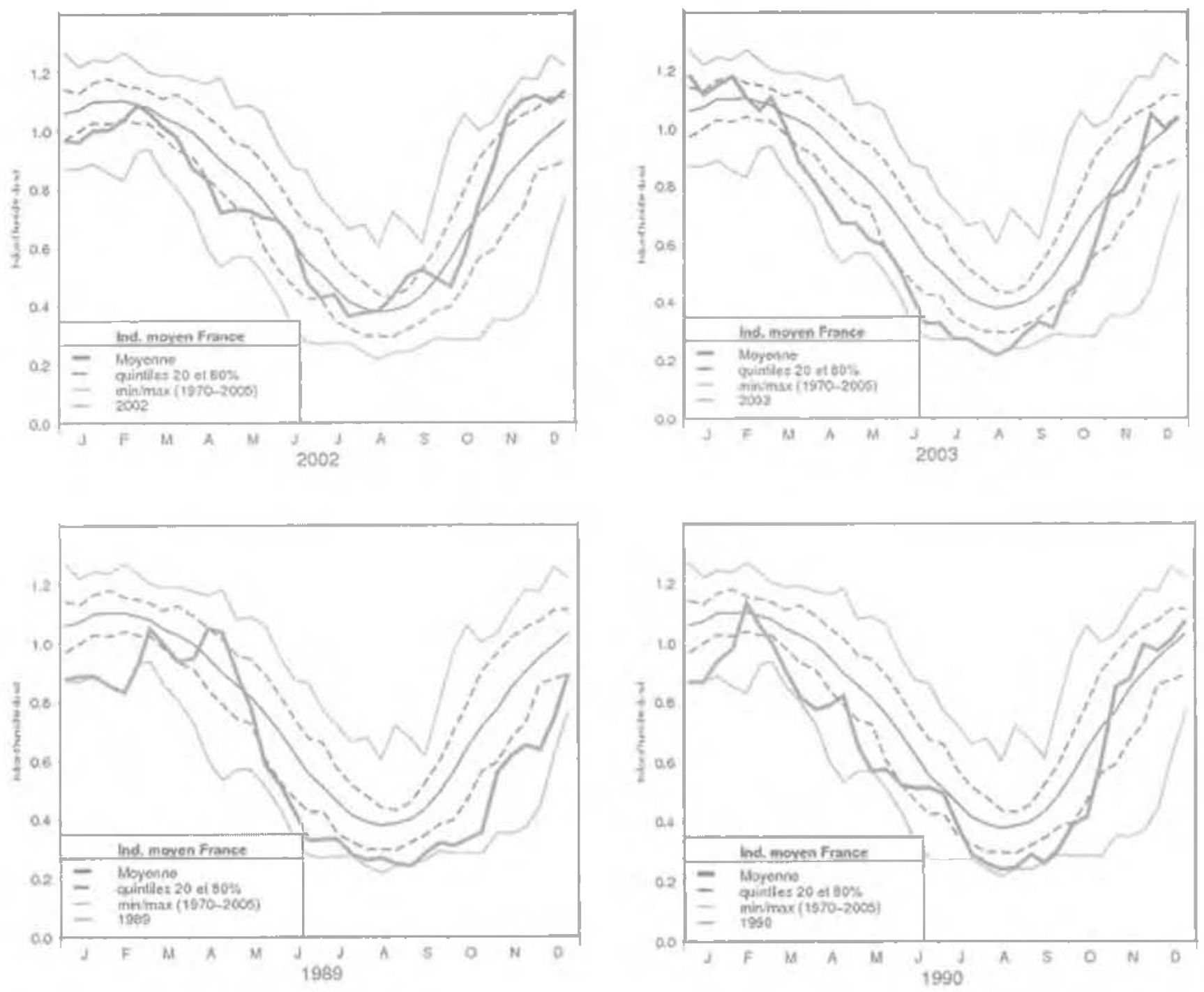

FG.9 Suivi opérationnel de l'indice d'humidité des sols en moyenne sur l'ensemble de la France.

Operational monitoring of Soil Wetness Index over France and comparison to the climatological statistics of SIM.

Les simulations climatiques prenant en compte les scénarios d'évolution des concentrations en gaz à effet de serre indiquent pour la fin du siècle une augrientation des précipitations hivernales sur le nord de la France, mais aussi une accentuation des périodes de sécheresses en été, particulièrement sur le sud. On assistera probablement en parallèle à une augmentation de la demande en eau, ce qui fait que la question des ressources en eau deviendra cruciale. Des études d'impact du changement climatique sur la ressource en eau de grands bassins versants français ont été menées. Pour les bassins versants montagneux comme le Rhône et la Garonne, l'élévation de la température conduira certainement à une fonte du manteau nègeux plus précoce, ce qui aura pour conséquence de prolonger la période de basses eau, (Etchevers et al,, 2002 ; Caballero et al., 2007). Sur le bassin versant de la Seine, la situation estivale dépendra essentiellement de la recharge des nappes dans la période hivernale
(Ducharne et al., 2007). Ces études d'impacts seront poursuivies et développées dans le cadre de programmes de recherches en cours, comme le projet HyMeX (Hydrological cycle in the Mediterranean Experiment), centré sur la zone méditerranéenne.

Dans sa version actuelle, la chaine Safran-IsbaModcou utitisée dans la version d'ISBA opérationnelle (deux températures et trois réservoirs pour l'hydrolagie dans le sol], n’est pas la version la plus adaptée au problème posé. On espère, à l'avenir utiliser une version plus évoluée (version multicouche), qui devrait être mieux à même de reproduire les phénomènes à prendre en compte (transferts verticaux, gradients de texture, extraction racinaire). Cette nouvelle version devra être construite et validée grâce aux données et modèles détaillés existants, comme ceux du projet ARGIC, et grâce à de nouveaux projets à mettre en place avec les équipes ayant développé un savoir-faire sur ce sujet. 


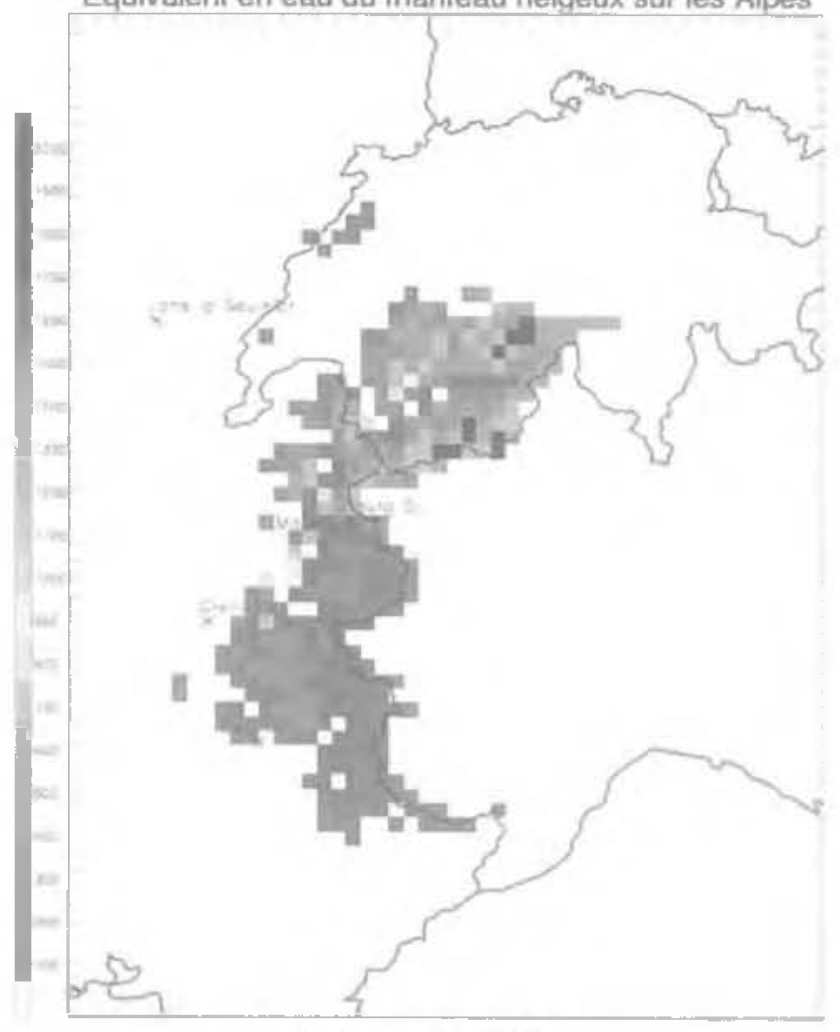

Le 1er avril 2006
Equivalent en eau du manteau neigeux sur les Alpes

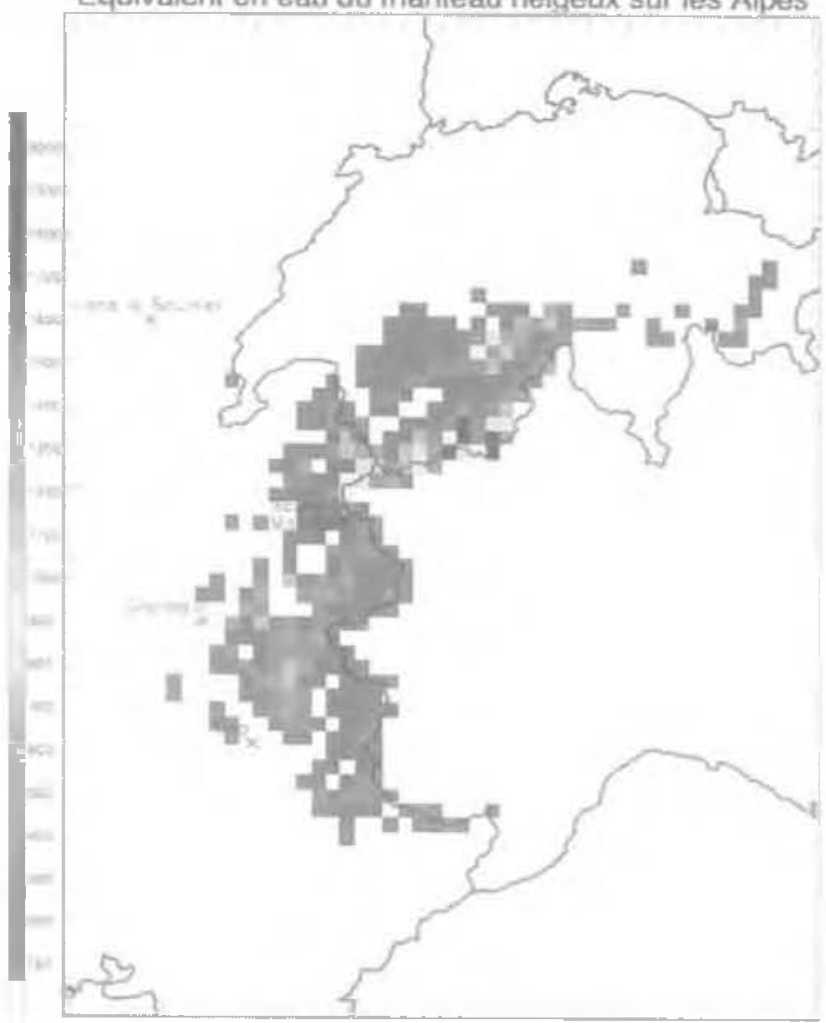

Le 1er avril (1995-2005)

Fa. 10 Contribution de Météo-France à la rubrique manteau neigeux du BSH national : (a) équivalent en eau du manteau neigeux sur les Alpes au $1^{\mathrm{ar}}$ avril $2006\left(\mathrm{~kg} / \mathrm{m}^{2} / \mathrm{s}\right)$; (b) moyenne de l'équivalent en eau du manteau neigeux au $1^{\mathrm{er}}$ avril sur la période $1995-2005$.

Contribution of Mété-France to the colurn snow-COvered coat of the national BSH: (a) snow water equivalent over the Aps on April 1st. $2006\left(\mathrm{~kg} / \mathrm{m}^{2} / \mathrm{s}\right)$; (b) average of the snow water equivalent over the Alps on April 1st over the period $1995-2005$

\section{Bibliographie}

Boone A. Calvet J.C. Noilhan J, - lrclusion of a third layer in a land surface scheme using the force restore. J. Appl Meteor, 38(11), 1999, p. $1611-1630$.

Brochet - Aspects climatiques de la sécheresse 1976. La Météorologie, 6e série. $\mathrm{r}^{\circ} 7$, décembre 1976.

Caballero Y. Morel S. Habets F. Noilhan J., Le Moigne P., Lehenaff A. Boone A - Hydrological sensitivity of the AdourGaronne river basin to climate change. Water Ressources Research, 2007.

Ducharme A, Baubion C. Begudoin N, Benoit M, Billen G. Brisson N, Garnier J, Kieken H, Lebonvallet S, Ledoux E, Mary B, Mignolet C. Poux X, Sauboua E, Schot C, Théry S, Viennot P. - Long term prospective of the Seine river sys tem: Confronting climatic and direct anthropogenic changes. Science of the Total Environment, vol. 375, Issues 1-3 2007, p 292-311

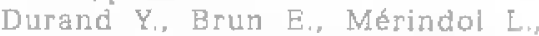
Guyomarc $h$ G., Lesaffre B., Martin E. . A meteorological estimation of relevant parameters for snow models. Annales Geophysicae, 18, 1992, p. 65-71.

Etchevers P., C. Golaz, Habets F - Simulation of the water budget and the river flows of the Rhone basin from 1981 to 1994. J. Hydrol. 244, 2001, p. 60-85.
Etchevers P., Golaz C., Habets F., Noilhan J. - Impact of climate change on the Rhone river catchment hydrology. Journal of Geophysical Pesearch, $107, \pi^{\circ} \mathrm{D} 16$, 10.1029/2001JD000490, 2002, p. 1-18.

Habets $F_{*}$ Etchevers $P_{\text {. }}$ Golaz C. Leblois E., Ledoux E., Martin E., Noilhan I. Ottle C - Simulation of the water budget and the river flows of the Rhone basin. Journal of Geophysical' Research, vol. 104, 1999, p. 31145-31172.

Ilabets F, Boone A, Champeaux J.-L., Ftchevers, P. Franchictéguy L.. Lebiois E. Ledoux E., LeMoigne P, Martin E., Morel 5., Noilhan J., Quintana Segtui P. Rousset Regimbeau $F$, Viennot P. - The SafrarIsba-Modcou hydrometeorological model applied ower France. Soumis at Journal of Geophysical Research, 2007

Jacquart C., Choisnel E. Un modèle de bilan hydrique simplifié à deux réservoirs utilisables en agrométéorologie. La Météorologie, g" série, $\mathrm{H}^{2} \mathrm{~g}, 1995$.

Ledoux E., Girard G., De Marsily G. Deschenes J. - Spatially distributed modeling: Conceptual apporach, coupling strface water and ground-water. Unsaturated flow bydrologic modeling: theory and practice, H. J. Morel-Seytoux (ed.), 1989, p 434-454, NATO Sciences Service.
Masson V., Charnpeaux J.-L., Chauvin F. Meriguer C. Lacaze R. - A global dato base of land surface parameters at $1 \mathrm{~km}$ resolution in meteorological and climate models. Jowmal of Climatology, 16, 2003, p. 1261-1282.

Noilhan J., Planton S. - A simple param eterization of and surface processes for metorological models. Monthly Weather Review, 117, 1989, p. 536-549

Noilhan I.. Mahfouf J.F.- The ISBA land surface parameterization scheme. Glabal Planetary Change, 13, 1996, p. 145159.

Quirtana Segui P., Le Moigne P. Durand $Y_{.}$, Martin E. Habets $F$, Baillon $M$. Canellas G. Franchistéguy L., Morel S. - Analysis of near surface abrnospheric variables: validation of the Safrarn analysis over France. Journal of Applied Meteorology and Gimatology, 2007.

Voirin-Morel, S. Modélisation distribuée des flux d'eau et d'énervie et des débits à l'échelle régionale du bassin Adour Garonne. PhD Thesis, université Toulouse III, Toulouse, France, 2003 (http// www.cig.ensmp. fr/-hydro/T HE/the. htm?. 